\title{
Positioning control of square arrangements of solar panels by solar tracing using fuzzy logic
}

\section{Control de posicionamiento de arreglos cuádruples de paneles solares por seguimiento solar utilizando lógica fuzzy}

\author{
DOI: $10.46932 / \mathrm{sfjdv2n2-168}$
}

Received in: March 1st, 2021

Accepted in: May 30th, 2021

\author{
MSc. Diego Armando Mejía \\ Universidad de Pamplona \\ Ciudadela Universitaria. Pamplona, Norte de Santander, Colombia. \\ E-mail: diego.mejia@unipamplona.edu.co \\ PhD. Ivaldo Torres Chávez \\ Universidad de Pamplona \\ Ciudadela Universitaria. Pamplona, Norte de Santander, Colombia. \\ E-mail: ivaldo.torres@unipamplona.edu.co \\ MS(c). Abelardo Mejía \\ Universidad de Pamplona \\ Ciudadela Universitaria. Pamplona, Norte de Santander, Colombia. \\ E-mail: abelardo.mejia@unipamplona.edu.co
}

\begin{abstract}
In this project a fuzzy control system is developed to follow the sun with its respective dynamics. The positioning of the panel must take into account changes in the weather, the disturbances that may occur, the area in which the tracker is located and so on. To do this, the maximum capture point positioning control system is studied using fuzzy logic. Prior to design and implementation, corresponding design criteria were established in instrumentation, mechanical structure, control, communication, and processing for prototype development. By means of the above, the photovoltaic array positioning system is designed, to then carry out the efficient implementation thanks to the calculations and adjustments made. After having the physical structure, we start to develop the positioning control of quadruple arrangements of solar panels with two degrees of freedom per maximum point of capture of solar radiation using fuzzy control, taking into account that the radiation sensors give a reference point. for the setpoint in the control loop and after this, control signals are sent to the selected actuators. Finally, the control system is validated for the positioning of the tracker with the fuzzy control loop implemented; In addition, the system is compared with the strategy implemented before a static system in order to analyze its efficiency.

In order for the solar tracking system to show greater efficiency compared to the static system, radiation sensors are implemented that compensate for the influence of climatic disturbances that any solar panel tends to suffer.
\end{abstract}

Keywords: Fuzzy control, quadruple arrays, solar traker, solar incidence.

\section{RESUMEN}

En este proyecto se desarrolla un sistema de control fuzzy para seguir el sol con su respectiva dinámica. El posicionamiento del panel debe tener en cuenta los cambios del clima, las perturbaciones que se pueden 
presentar, la zona en la que se encuentra el seguidor y demás. Para ello, se estudia el sistema de control de posicionamiento por máximo punto de captura utilizando lógica difusa. Antes de realizar el diseño e implementación, se establecieron los criterios de diseño correspondientes en la instrumentación, la estructura mecánica, el control, la comunicación y el procesamiento para el desarrollo del prototipo. Por medio de lo anterior, se diseña el sistema de posicionamiento de arreglos fotovoltaicos, para luego realizar la implementación eficiente gracias a los cálculos y ajustes realizados. Luego de tener la estructura física, se parte a desarrollar el control de posicionamiento de arreglos cuádruples de paneles solares con dos grados de libertad por máximo punto de captura de radiación solar, utilizando control fuzzy, teniendo en cuenta que los sensores de radiación dan un punto de referencia para el setpoint en el lazo de control y posterior a esto se envían señales de control a los actuadores seleccionados. Finalmente se hace la validación del sistema de control para el posicionamiento del seguidor con el lazo de control fuzzy implementado; además, se compara el sistema con la estrategia implementada ante un sistema estático con el fin de analizar su eficiencia.

Para que el sistema de seguimiento solar muestre una mayor eficiencia ante el sistema estático se implementan sensores de radiación que compensan la influencia de las perturbaciones climáticas que tiende a sufrir cualquier panel solar.

Palabras clave: Control fuzzy, arreglos cuádruples, seguidor solar, incidencia solar.

\section{INTRODUCION}

En la búsqueda de fuentes de energías alternativas que ayuden a proteger el medio

ambiente, los Gobiernos contemporáneos han tomado la decisión de utilizar el Sol como una gran fuente de energía ya que es infinita e inagotable.

Con el fin de aumentar la popularidad de la energía solar fotovoltaica se está trabajando en el aumento de su uso eficiente (Curtin y Magolis, 2009). La energía solar presenta un gran inconveniente en cuanto a la dificultad de su introducción al mercado eléctrico debido al elevado costo MWh, sin embargo, se estima que se presentará una reducción del 18\% cada que la producción se duplique. Las diferentes tecnologías de fabricación, así como la producción a gran escala han contribuido de manera significativa a la reducción de los precios de los módulos fotovoltaicos (Informe de Ecologistas en Acción, 2005).

Por tal motivo resulta imprescindible maximizar la captura de irradiancia, ya que esto permite maximizar la eficiencia en el sistema fotovoltaico. Si bien, a nivel teórico, el problema de seguimiento estaría resuelto aplicando las ecuaciones solares (conociendo las coordenadas de la instalación y la hora solar, se podría obtener la dirección de apuntamiento y aplicar una estrategia de seguimiento en bucle abierto), en la práctica, en una instalación real, los resultados que se obtienen no son satisfactorios debido a una serie de incertidumbres (tipos de radiación, imprecisiones de montaje, holguras de mecanismos, deformaciones de la estructura, etc.) (Berenguel y Rubio F.R, 2004); que degradan el funcionamiento del sistema de seguimiento. 
Bajo estas consideraciones, se hace necesario complementar o sustituir la estrategia en bucle abierto, basada en ecuaciones solares, por otras estrategias más sofisticadas, en las que, de alguna forma, se pueda aplicar un esquema realimentado.

Este trabajo se basa en el desarrollo de un sistema seguidor solar de arreglos cuádruples bajo control fuzzy.

La mayor parte de la energía que llega a nuestro planeta procede del Sol, esta energía es emitida en forma de radiaciones electromagnética y se distingue por sus diferentes longitudes de onda, como son las radiaciones gamma, rayos X y ultra violeta con menor longitud de onda por lo cual llevan más energía; los infrarrojos y las ondas de radio que tienen mayor longitud de onda, de menos energía. Estas radiaciones llegan a la superficie del planeta como radiaciones directa, difusa y reflejada. La atmosfera es diatérmana por lo cual no se calienta directamente por la radiación solar sino de manera indirecta a través de la reflexión (suelo, mares y océanos).

La energía solar tiene longitudes de onda entre 0.15 y 4 micras por lo que puede ionizar un átomo, excitar electrones, disociar una molécula o hacerla vibrar por la radiación infrarroja provocándose un calentamiento en la atmósfera. Estas radiaciones son aprovechadas por las células fotovoltaicas elaboradas en silicio monocristalino, silicio amorfo, silicio demonocristalino, arseniuro de galio, policristalino. Al grupo de células fotoeléctricas para energía solar se le conoce como panel fotovoltaico, estos consisten en una red de células solares conectadas como circuito en serie para aumentar la tensión de salida hasta el valor nominal (usualmente se utilizan 12 o $24 \mathrm{~V}$ ) a la vez que se conectan varias redes como circuito paralelo para aumentar la corriente eléctrica que es capaz de proporcionar el dispositivo.

El objetivo de este proyecto es presentar el diseño e implementación de un prototipo para el posicionamiento con dos grados de libertad y control de arreglos cuádruples de paneles solares por control de máximo punto de captura por lógica fuzzy, con el fin de incrementar el aprovechamiento de la energía solar. El dispositivo utilizado permite aumentar la radiación captada por el módulo solar mediante el movimiento continuo del panel. Tal movimiento busca mantener toda el área del panel en posición perpendicular a los rayos del sol. El prototipo presentado permite mejorar el rendimiento de una instalación fotovoltaica (Celia y Labrada, 2019).

\section{SEGUIDOR SOLAR COMO DISPOSITIVO DE OPTIMIZACIÓN}

En los sistemas fotovoltaicos existe la posibilidad de implementar un dispositivo adicional con el fin de aumentar la captación de radiación solar y por ende la energía suministrada por la instalación, tal dispositivo es un seguidor solar. 
Un seguidor solar es un dispositivo conformado básicamente por una parte fija y una móvil, cuya finalidad es el aumento de la captación de radiación solar, para lo cual cuenta con una superficie de captación que debe permanecer perpendicular a los rayos del sol durante el día y dentro de su rango de movimiento (Celia y Labrada, 2019).

Los seguidores solares pueden ser clasificados según el tipo de movimiento que realicen y según el algoritmo de seguimiento.

\subsection{SEGUIDOR SOLAR SEGÚN EL TIPO DE MOVIMIENTO.}

\subsubsection{Seguidor de un solo eje}

La rotación de la superficie de captación se hace sobre un solo eje, este puede ser horizontal, vertical u oblicuo. Este tipo de seguimiento representa un mínimo grado de complejidad y su costo es menor en comparación con otros tipos de seguidores. La limitación de este tipo de seguidor consiste en que no puede realizar un seguimiento completo del sol ya que solo puede seguir el azimut o la inclinación solar, pero no ambas (Departamento Nacional de Planeación Subdirección Territorial y de Inversiones Públicas, 2016).

\subsubsection{Seguidor de dos ejes:}

Este tipo de seguidor cuenta con dos grados de libertad y está en capacidad de realizar un seguimiento total del sol, tanto en inclinación como en azimut, aunque el rendimiento de la instalación puede ser superior en comparación con los de un solo eje, es importante mencionar que el de dos ejes resulta ser más costoso.

\subsection{SEGUIDOR SOLAR SEGÚN EL TIPO DE ALGORITMO DE SEGUIMIENTO.}

\subsubsection{Según la luminosidad:}

El algoritmo de este tipo de seguidor basa su funcionamiento en la señal entregada por uno o varios sensores, dependiendo de dicha señal se envía un comando de control a uno o varios motores para que se posicionen en el punto más adecuado de luminosidad. Este tipo de seguimiento resulta ser de fácil implementación.

\subsubsection{Con programación astronómica:}

Este tipo de seguidor presenta una total independencia de las condiciones climáticas ya que su algoritmo no requiere de sensores que indiquen cual es el punto más luminoso. El seguimiento en este caso depende únicamente de una serie de ecuaciones que predicen la ubicación del sol en cualquier 
momento. Aunque este tipo de seguidor resulta ser de gran precisión cabe mencionar que la implementación del algoritmo representa un alto grado de complejidad (Departamento Nacional de Planeación Subdirección Territorial y de Inversiones Públicas, 2016).

\section{DESCRIPCIÓN DEL SEGUIDOR DISEÑADO}

El seguidor solar se puede dividir en las siguientes partes: estructura mecánica (fija y móvil), equipos de generación y transformación de la energía producida, equipos de control de los movimientos de orientación y equipos de instrumentación específica.

La estructura mecánica tiene dos grados de libertad, uno para seguir el movimiento del Sol en azimut y otro para seguirlo en elevación. La Figura 1 muestra el seguidor solar real utilizado

en el presente trabajo. El seguidor dispone de una superficie de captación total de 6,51833 Mts².

Figura 1: Seguidor solar con paneles (figura del prototipo).

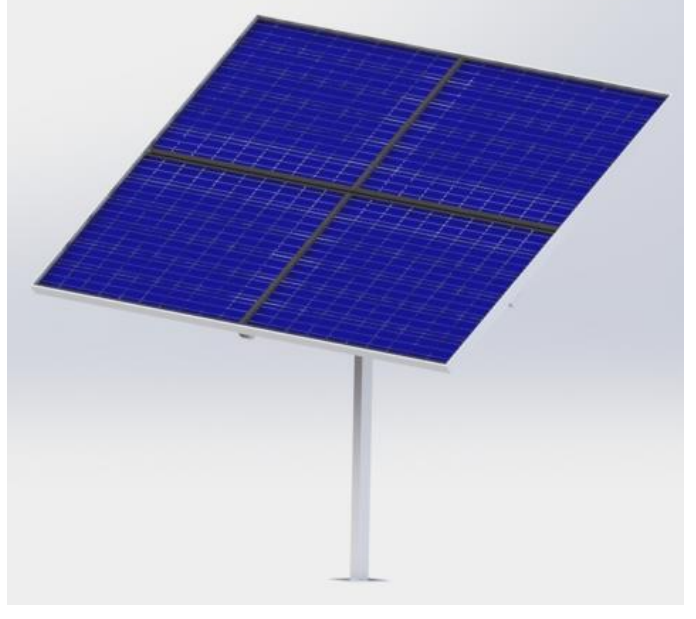

Para orientar el sistema, se dispone de dos actuadores lineales de $2500 \mathrm{~N}$ comandados por dos drivers de motor Monster VNH2SP30 gestionados desde una tarjeta DAQ 6009, como se ilustra en la figura 2.

Figura 2: Arquitectura electrónica del sistema.

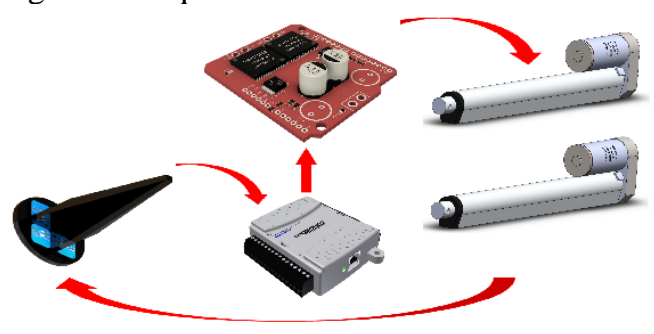


Los equipos generadores serán el conjunto de paneles solares. La instalación dispone de 4 paneles solares modelo Exe Modelo EXP 260 /156-60.

Como equipo de control principal se usó una tarjeta DAQ 6009 de National Instruments. El algoritmo de control determina cuándo y a qué velocidad se deben mover los actuadores lineales, generando las correspondientes referencias a los drivers motor Monster VNH2SP30.

Se utilizan además un medidor de potencia instantánea generada por la instalación en el lado de corriente continua y cuatro sensores de radiación solar que proporcionan los setpoint para los ángulos de orientación. así como los valores de irradiancia solar normal directa.

\section{ALGORITMO DE SEGUIMIENTO.}

El seguidor descrito en este artículo en cuanto al tipo de movimiento pertenece a la clasificación de seguidor de dos ejes. Según el tipo de algoritmo, corresponde al seguidor de una fuente luminosa.

El dispositivo seguidor consta de cuatro etapas básicas:

- Recepción y acondicionamiento de la señal de entrada.

- Procesamiento de la señal.

- Etapa de control fuzzy.

- Etapa de potencia.

En la Figura 3 se muestra el esquema básico correspondiente al prototipo de seguidor solar desarrollado.

Figura 3. Esquema del prototipo de seguidor solar.

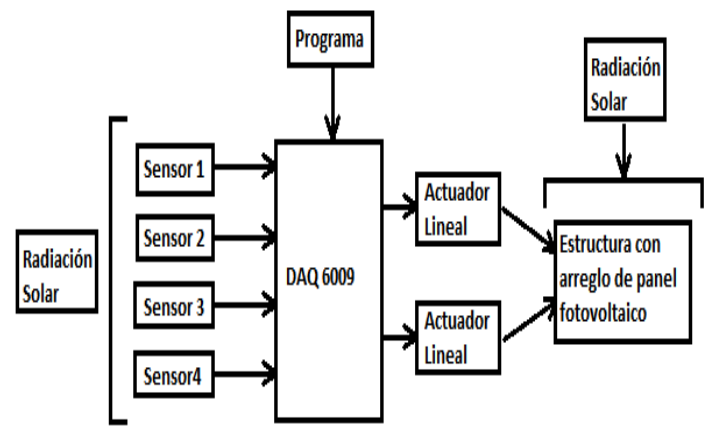

En la etapa de recepción y acondicionamiento de la señal, se hace uso de cuatro sensores y la tarjeta de adquisición DAQ 6009, el tiempo de muestreo es de 10 ms. Los sensores UVM30A son dispuestos de tal forma que los voltajes sean iguales cuando los rayos luminosos incidan de manera perpendicular sobre la superficie colectora. 
La etapa de procesamiento de la señal consiste básicamente en realizar una comparación entre los valores obtenidos de los cuatro sensores UVM30A.

Definiendo la señal del sensor uno como S1, del sensor dos como S2, del sensor tres como S3 y del sensor cuatro como S4, agrupándolos de a pares se pueden presentar tres posibles casos: que S1>S2 o que $\mathrm{S} 1<\mathrm{S} 2$ y finalmente que $\mathrm{S} 1=\mathrm{S} 2$ y que $\mathrm{S} 3>\mathrm{S} 4$ o que $\mathrm{S} 3<\mathrm{S} 4$ y finalmente que $\mathrm{S} 3=\mathrm{S} 4$.

Dependiendo de los casos que se hayan desarrollado en el sistema de control fuzzy, se definirá un sentido de giro para los actuadores lineales. En el caso en que S1 y S2 o S3 y S4 sean iguales, los motores deberán permanecer inmóviles. Este proceso corresponde a la etapa de control.

En cuanto al sensor el cual da referencias de los ángulos azimutal y de elevación, consta de una estructura la cual agrupa por pares a los cuatro sensores y se encarga de generar sombras, como se aprecia en la figura 4, para que el sistema tenga una señal de referencia para mover sus actuadores.

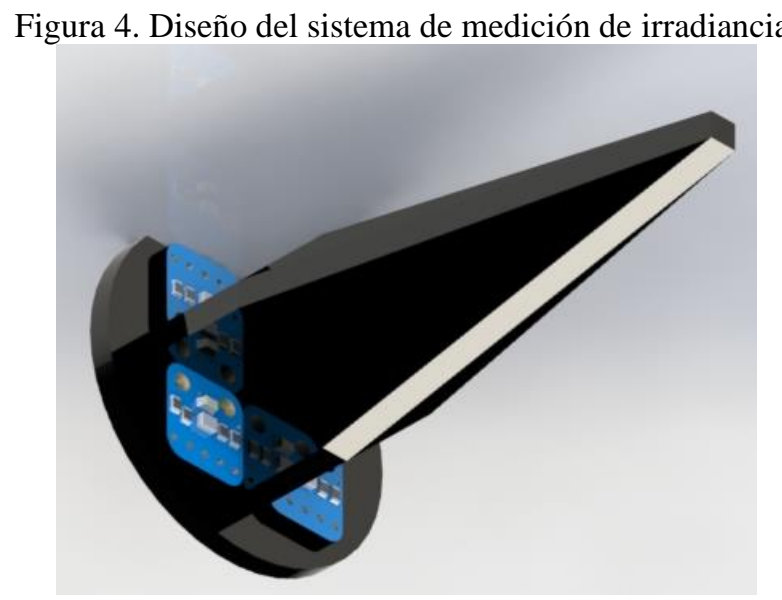

La colocación del sensor se realiza de tal forma que por pares puedan enviar la señal de referencia al sistema de control como se observa en la figura 5.

Figura 5. Distribución de sensores de irradiancia.

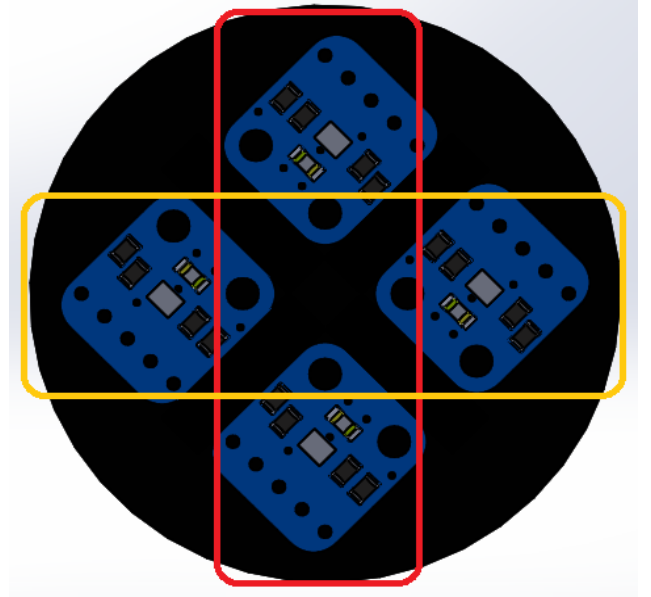


La etapa de potencia está conformada por dos actuadores lineales de $2500 \mathrm{~N}$ de 12 voltios, cuya función es mover la superficie de captación según se defina en la etapa de control.

Figura 6. Diseño de un sistema con control difuso.

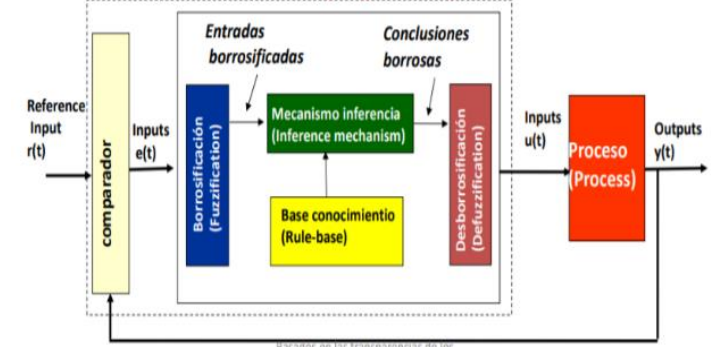

Figura 7. Diagrama en bloque del lazo de control por lógica difusa.

Sistemas de Seguimiento Solar en Lazo Cerrado.

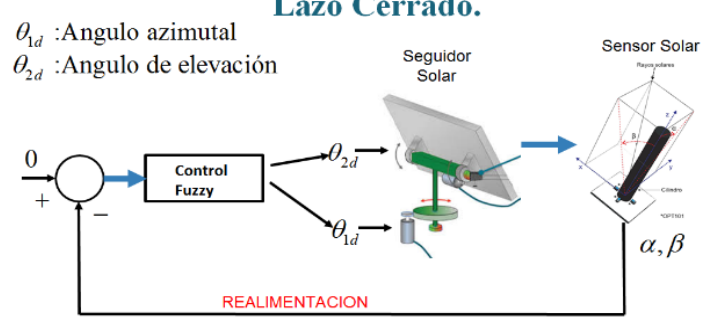

La programación del control fuzzy para el seguidor se realiza mediante el software de Labview a través de la tarjeta DAQ 6009. En la figura 8 se muestra una fracción del código correspondiente a la captura de señales, procesamiento por medio del controlador difuso y la actualización de la salida para los actuadores lineales.

Figura 8. Código del control fuzzy en Labview.

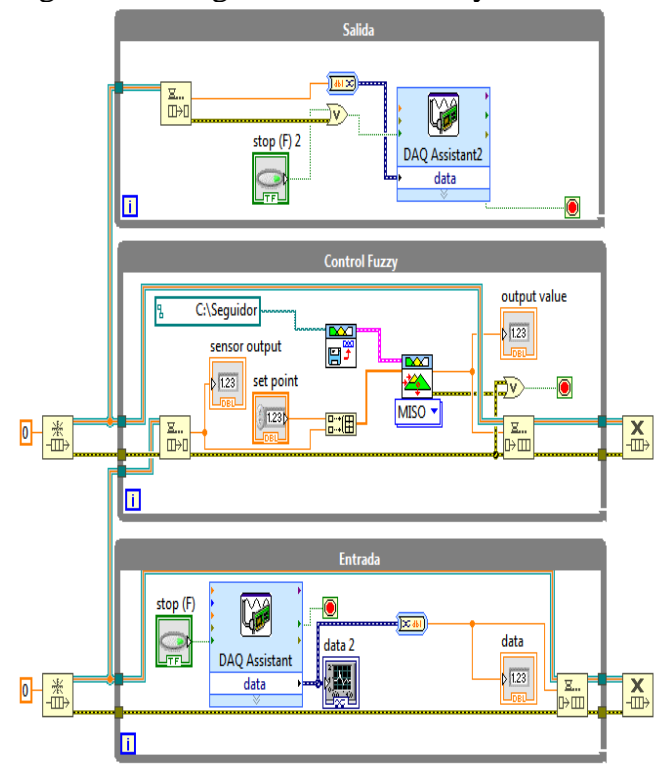


En la Figura 9 se observa la representación del conjunto difuso de las entradas S1,S2,S3,S4 y las salidas posición M1, posición M2, donde a cada valor de [0 600], le corresponde un rango [0 1] según sea su pertenencia. Cuando el sistema queda estabilizado en "OK", el sistema de seguimiento recibe en su entrada la misma cantidad de voltaje entre los sensores y por lo tanto la celda fotovoltaica está posicionada correctamente. Los conjunos difusos de salida que se muestran en la figura 8, son similares para los motores 1 y 2, debido a que ambos comparten las mismas características mecánicas y eléctricas; además de recibir la información por parte de sensores con el mismo comportamiento.

Figura 9. Conjunto difuso de entradas y salidas.

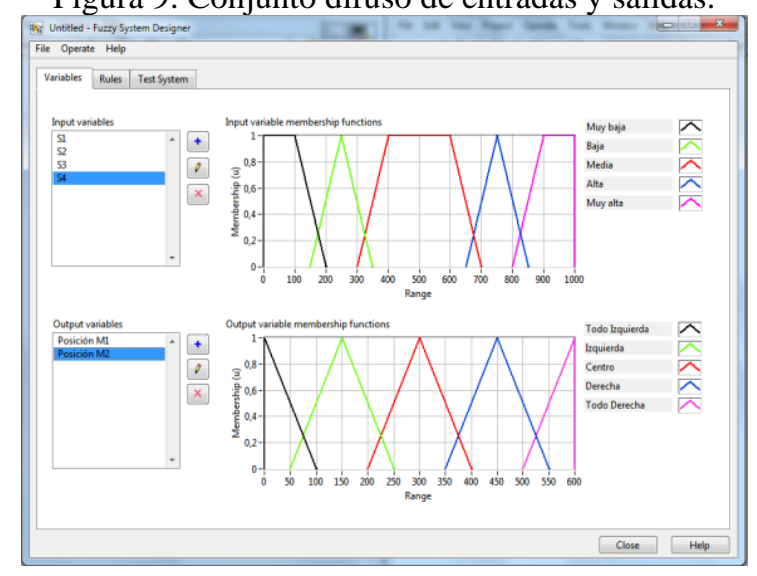

En la tabla 1 se muestran las reglas de control difuso asociadas a un motor del sistema. En total son 25 reglas por cada actuador, cada una muestra la relación existente entre los conjuntos difusos que se creados y su nivel de dependencia, es decir que se establecen reglas donde el sistema difuso se encargara de crear las salidas adecuadas para cada caso de los conjuntos difusos.

Tabla 1. Tabla de reglas para los conjuntos difusos.

\begin{tabular}{c|lllll}
\hline S1,S3 & & & & & \\
S2,S4 & $\begin{array}{c}\text { Muy } \\
\text { baja }\end{array}$ & Baja & Media & Alta & $\begin{array}{c}\text { Muy } \\
\text { alta }\end{array}$ \\
\hline Muy baja & C & I & I & TI & TI \\
Baja & D & C & I & TI & TI \\
Media & D & D & C & I & I \\
Alta & TD & TD & D & C & I \\
Muy alta & TD & TD & TD & D & C \\
\hline
\end{tabular}

C: Centro. D: Derecha. I: Izquierda. TD: Todo Derecha. TI: Todo Izquierda.

\section{PRUEBAS REALIZADAS Y RESULTADOS OBTENIDOS}

Las pruebas para verificar el correcto funcionamiento del dispositivo seguidor solar con el sistema de control fuzzy se realizaron en campo bajo la fuente solar, sobre un prototipo de una instalación fotovoltaica cuyo esquema se puede observar en la figura 10. 
Figura 10. Sistema fotovoltaico cuádruple.

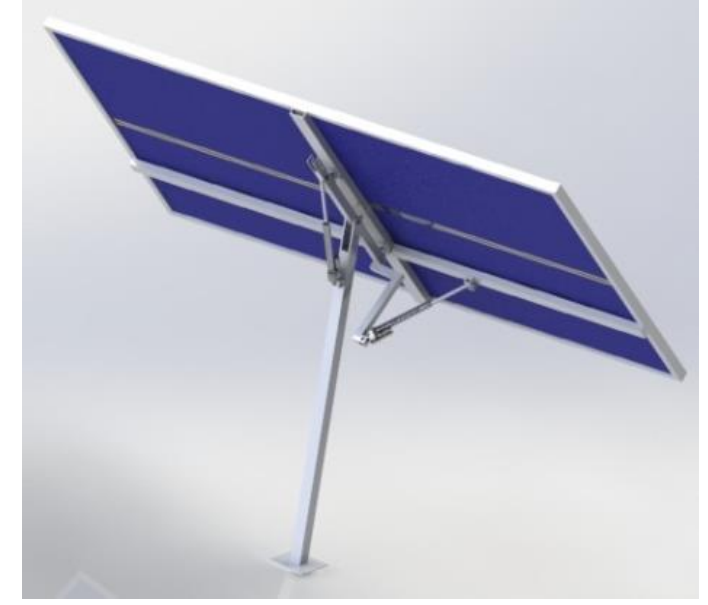

Utilizando el arreglo fotovoltaico cuádruple estático y posteriormente con la estructura y el sistema de control fuzzy se tomaron datos de potencia de salida del panel fotovoltaico, como se observa en la figura 11, en esta ocasión haciendo uso de una tarjeta de adquisicion de datos National instruments 6009, los resultados obtenidos con esta tarjeta se graficaron en Excel, directamente mediante el uso de la interfaz gráfica de labview.

Figura 11. Potencia entregada por el arreglo fotovoltaico en un día por el sistema estático y aplicando control fuzzy al seguidor.

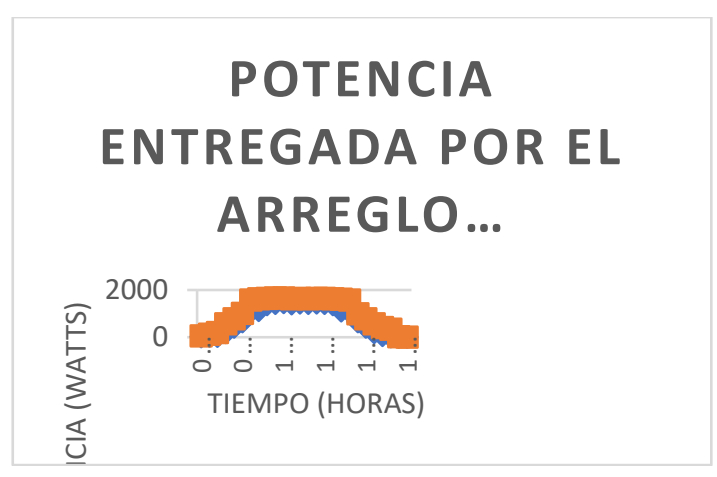

Al integrar la curva de potencia entregada por el arreglo fotovoltaico en un día por el sistema estático, se obtiene la potencia producida de $9963 \mathrm{WH} /$ día, mientras que al integrar la curva de potencia entregada por el arreglo fotovoltaico en un día aplicado el control fuzzy al sistema de seguimiento solar se obtiene 13443 WH/día. El sistema es capaz de maximizar la captura de irradiancia mejorando así la eficiencia. 
Figura 12. Radiación solar capturada en un día por el sistema fotovoltaico estático y por el sistema seguidor solar con control fuzzy.

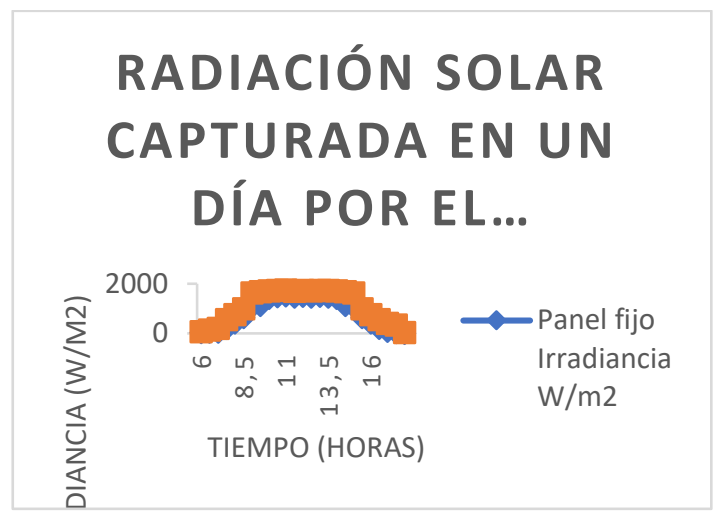

Analizando los datos obtenidos de la figura 12, el sistema seguidor solar con control fuzzy ampliza y mantiene estable la captura de irradiancia entre las nueve de la mañana y las cuatro de la tarde y a su vez aprovecha la irradiancia de las horas de la mañana, mientras que el sistema de arreglos fotovoltaico estatico no aprovecha la irradiancia de las horas de la mañana (6-9 AM) y las horas de la tarde (4-6 PM), haciendo que el sistema sea menos eficiente.

El comportamiento de los dos sistemas es muy parecido entre las 9 AM y las 4 PM, con la diferencia de que la proporcionalidad de la irradiancia caturada por el sitema controlado, siempre es mayor a la del sistema estático, esto se debe a que el sistema de control puede recoger irradianza difuza, refleja y la directa mientras que el sistema estático no tiene esta caracteristica.

\section{CONCLUSIONES}

El prototipo construido cumple con las características requeridas de seguimiento en dos ejes, realizando el seguimiento azimutal y ángulo de elevación del sol.

El sistema presenta una alta resolución de entrada que le permite muestrear de forma satisfactoria la señal de voltaje proveniente de los sensores y de esta manera reaccionar adecuadamente ante mínimos cambios de irradiancia.

Sistema de control desarrollado puede ser utilizado en otras aplicaciones que requieran una actividad de seguimiento solar, como colectores solares, hornos solares, entre otros.

Se desarrolló un análisis, con los resultados de las pruebas realizadas tanto con el sistema cuádruple de paneles solares estático, como con el control de posicionamiento de arreglos cuádruples de paneles solares por seguimiento solar utilizando lógica fuzzy, observando que se genera mayor potencia de salida con el sistema de control fuzzy, esto se puede observar en la figuras 10, con ayuda de los datos obtenidos mediante las pruebas también se pudo determinar que la eficiencia máxima para captar los rayos solares se da con el sistema de posicionamiento aplicando el control fuzzy. 
Otro punto a considerar es la ventaja del control fuzzy ante otros controladores los cuales no son orientados a sistemas donde se necesita inversión de giro como lo es en este caso, ya que la estructura presenta dos actuadores lineales. 


\section{REFERENCIAS}

Abu-Khader, M. M.; Badran, O. O. and Abdallah, S. 2008. Evaluating multi-axes sun-tracking system at different modes of operation in Jordan. Renewable and sustainable energy reviews. 12: 864-873.

Arroyo Romero, G. y Cortés Montes de Oca, L. A. (2015). Diseño y construcción de un mecanismo Automatizado para el seguimiento de la trayectoria solar (tesis de pregrado). Universidad Nacional Autónoma de México, Ciudad de México, México.

Ahmet Senpinar, M. C. (2012). Evaluation of power output for fixed and two-axis tracking PVarrays. Applied Energy 92(1), 677-685.

Berenguel M., Rubio F.R. (2004). An artificial vision-based control system for automatic heliostat positioning offset correction in a central receiver solar power plant, Sol. Energ., vol. 76, pp 563-75.

Blanco-Muriel M., Alarcón-Padilla D.C. (2001) Computing the solar vector, Solar Energy, vol. 70, pp 431-441.

Celia E. Labrada R, Miriam M. (2019). DISEÑO DE LA COLOCACIÓN DE PANELES SOLARES FOTOVOLTAICO PARA MAYOR CAPTACIÓN DE ENERGÍA. Pistas Educativas, No. 134, México, Tecnológico Nacional de México en Celaya

Fernández F.E., Rodrigo P. (2013) Models for the electrical characterization of high concentration photovoltaic cells and modules: A review, Renewable and Sustainable Energy Reviews, vol. 26, pp. 752760 .

J. F Curtin, J. Magolis. (2009). El Uso Eficiente de la Energia, Departamento de Estado de Estados Unidos, Oficina de Programa de información Internacional, vol 14,numero 4.

Informe de Ecologistas en Acción. Propuesta Para El Desarrollo de la Energía Solar Fotovoltaica, Mayo de 2005.

MASICASALS, Orestes, Sistemas difusos dinamicos para el tratamiento de información temporal imprecisa, Universidad Poltecnica de Cataluña, Departamento de Teoria de las Señales y Comunicaciones, Barcelona, 1997.

NATIONAL Instruments Corporation, "PID and Fuzzy Logic Toolkit", Manuel de usuario, junio 2019. Noa-Diéguez, L. Y., Álvarez-Sánchez, V. y Pérez-Rodríguez, R. (2015). Diseño paramétrico asistido por computadora de un seguidor solar fotovoltaico a un eje polar. Ciencias Holguín, XXI(2), 1-12.

Rubio F.R., Ortega M.G. (2007) Application of new control strategy for sun tracking, Energy Conversion and Management, vol. 48, pp. 2174-2184.

Yousef, H. A. 1999. Design and implementation of a fuzzy logic computer controlled sun tracking system. Proceedings of the IEEE International Symposium on Industrial Electronics. 3:1030-1034.

\section{SITIOS WEB}

Departamento Nacional de Planeación Subdirección Territorial y de Inversiones Públicas. Instalación de sistemas solares fotovoltaicos individuales en zonas no 
interconectadas.https://proyectostipo.dnp.gov.co/images/pdf/Celdas/ptceldas.pdf, 2016. (4 de febrero de 2020). 